\title{
Diagnostic accuracy of mammography, ultrasonography and magnetic resonance imaging in the detection of intraductal spread of breast cancer following neoadjuvant chemotherapy
}

\author{
KEI KUBOTA ${ }^{1}$, YASUHIRO OGAWA ${ }^{1}$, AKIHITO NISHIOKA ${ }^{1}$, SHINJI KARIYA ${ }^{1}$, SATOSHI ITOH ${ }^{1}$, \\ YORIKO MURATA $^{1}$, NORIHIKO HAMADA ${ }^{1}$, HIRONORI MAEDA ${ }^{2}$ and YOHSUKE TANAKA ${ }^{2}$ \\ Departments of ${ }^{1}$ Radiology and ${ }^{2}$ Surgery, Kochi University, Nankoku, Kochi 783-8505, Japan
}

Received September 18, 2006; Accepted November 30, 2006

\begin{abstract}
The purpose of this study is to evaluate the accuracy of mammography, ultrasonography, and contrast-enhanced magnetic resonance imaging for the diagnosis of intraductal spread of breast cancer following preoperative neoadjuvant chemotherapy. We evaluated a total of 168 areas of normal breast tissue outside the mass in 42 consecutive female patients with breast cancer using each imaging modality both before and after neoadjuvant chemotherapy. Neoadjuvant chemotherapy comprised two to four cycles of adriamycinbased CAF regimen. Multivariate analysis indicated that calcification on mammography and size of hypoechoic structures on ultrasonography prior to neoadjuvant chemotherapy shows a correlation with intraductal spread on pathologic study. Our study reveals that mammography and ultrasonography are useful in avoiding residual cancer cells caused by intraductal spread following conservative breast surgery.
\end{abstract}

\section{Introduction}

Breast conservation treatment (BCT) for breast cancer is a well-established therapy (1-10). Previous studies of patients with invasive ductal breast carcinoma treated with conservative surgery indicate that the presence of an extensive intraductal component in the excised specimen is strongly associated with subsequent breast cancer recurrence; this diminishes the patient's quality of life $(11,12)$. Preoperative detection of intraductal spread (IDS) is extremely important to avoid local recurrence. IDS of breast cancer can be identified using breast-imaging techniques such as mammography (MMG),

Correspondence to: Dr Kei Kubota, Department of Radiology, Kochi University, Oko-cho, Nankoku-shi, Kochi-Prefecture 783-8505, Japan

E-mail: kubotak@med.kochi-u.ac.jp

Key words: breast cancer, mammography, ultrasonography, magnetic resonance imaging, intraductal spread ultrasonography (US) and magnetic resonance imaging (MRI) (13-17).

Preoperative systemic neoadjuvant chemotherapy (NAC) improves both local control and survival rate $(1,3,4,10,18-24)$. To our knowledge, breast-imaging techniques have not been used to identify IDS in breast cancer following preoperative chemotherapy. In the current study, we investigate the ability of breast imaging techniques to detect IDS in breast cancer following NAC.

\section{Materials and methods}

This study considered 42 consecutive breast cancer patients who were candidates for BCT intensified with preoperative NAC $\left(600 \mathrm{mg} / \mathrm{m}^{2}\right.$ of cyclophosphamide, $20-40 \mathrm{mg} /$ body of pirarubicin, and $600 \mathrm{mg} / \mathrm{m}^{2}$ of 5-fluorouracil) between August 2000 and December 2005. All patients were female, with an average age of 51.5 years, range of 38-61 years. Surgery was performed in all 42 cases following NAC (2-4 cycles CAF regimen). The mean interval between the last administration of NAC and surgery was 21 days (range, 14-34 days). Breast cancer with absent or mild calcification outside the mass on MMG was resected with surrounding normal breast tissue in 37 cases ( 2 cases of quadrantectomy and 35 cases of partial resection with a macroscopically tumor-free margin of $2 \mathrm{~cm}$ ). For 5 cases of the 35 partially resected patients, additional resection was performed after concurrent rapid pathological analysis during surgery revealed the presence of cancer cells near the edge of the specimen. The remaining 5 cases with calcification throughout the majority of the breast on MMG underwent total glandectomy because this condition precludes tissue preservation according to BCT guidelines in Japan (25). Continuous pathologic specimens were obtained at 5-mm intervals to determine the existence of IDS. The breast tissue outside the mass was divided into four areas: the area closest to the nipple, the area furthest from the nipple, and the remaining two areas. The pathological results of these 168 areas from 42 patients were correlated with image findings of the three modalities.

Pre-NAC evaluation using the three modalities was performed within 14 days before the first administration of NAC. Post-NAC imaging examination was performed between the last NAC administration and surgery. 
$\mathbf{a}$

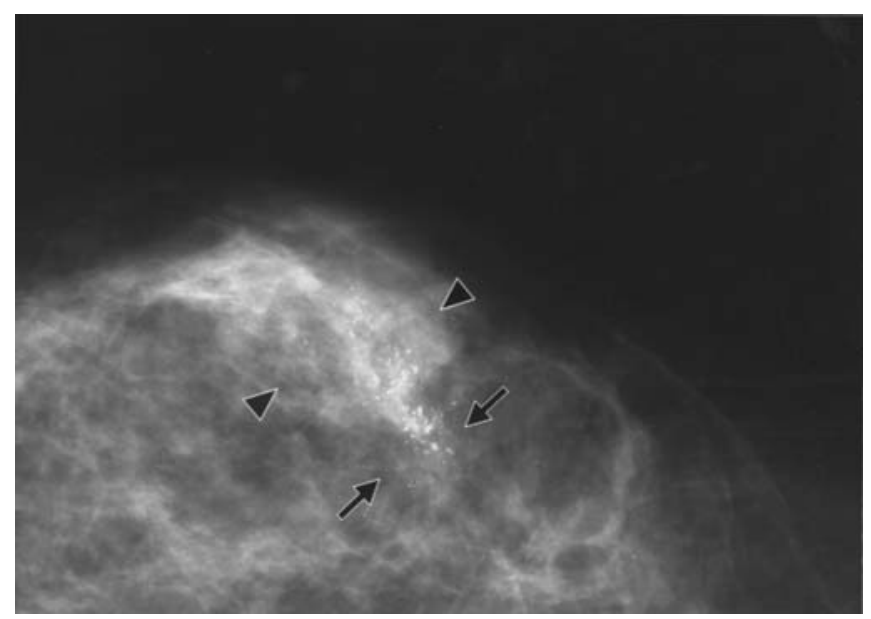

b

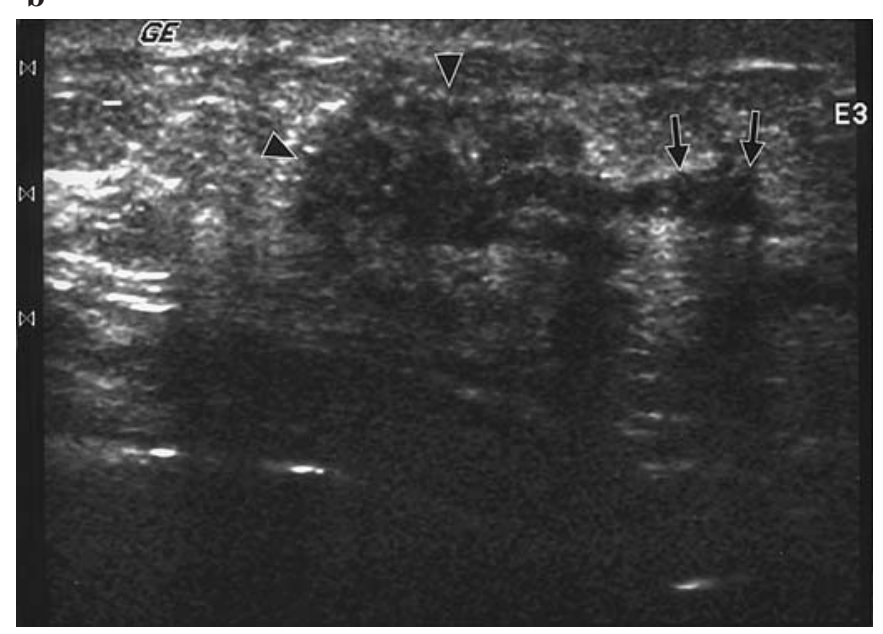

c

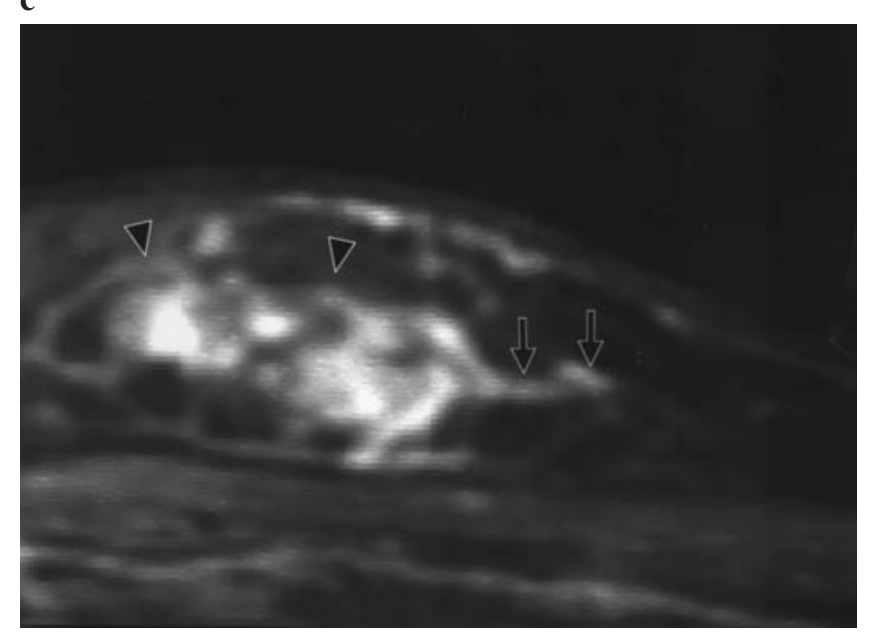

Figure 1. A 49-year-old woman with breast cancer. (a) MMG shows clusters of microcalcification (arrows) around the index tumor (arrowheads). (b) US depicts a hypoechoic structure (arrows) adjacent to the primary lesion (arrowhead). (c) Linear enhancement (arrows) outside the index tumor (arrowheads) is shown on MRI during the early phase of a contrast-enhanced study.

$M M G$. Bilateral MMG (craniocaudal and mediolateral-oblique view) was performed using the Senographe DS system (GE Medical Systems, Wilwaukee, WI) before and after NAC. Findings were recorded prospectively without the knowledge
Table I. Correlation between calcification on MMG and pathologic analysis of IDS.

\begin{tabular}{lrrrcr}
\hline & \multicolumn{2}{l}{ Calcification } & & \\
\cline { 2 - 3 } & + & - & & & P-value \\
\hline IDS & & & & Univariate & $<0.01$ \\
+ & 33 & 24 & 57 & Multivariate & $<0.01$ \\
\hline
\end{tabular}

Values represent the number of breast tissue areas.

of other imaging modality findings by one of several radiologists (Y.O., S.K. and A.N.) with 11-27 years of experience in $\mathrm{MMG}$ interpretation. Areas with microcalcification that were categorized as suspicious for cancer according to the BI-RADS lexicon (Fig. 1a) were considered to have IDS (26).

US. All patients were examined with ultrasound using a lineararray broadband transducer with a center frequency of 7$11 \mathrm{MHz}$ both before and after NAC (LOGIQ $700 \mathrm{MR}$, GE Medical Systems). US was performed by one of two radiologists (K.K. and I.S.) with 11-16 years experience who were blinded to the results of other imaging modalities. We closely analyzed the minor axis diameter of hypoechoic tubular or glandular tissue surrounding the main tumor during the execution of US (Fig. 1b). We used the greatest value of the minor diameter for analysis.

MRI. Dynamic MRI was performed using a 1.5-T unit (Signa Horizon, GE Medical Systems, Wilwaukee, WI). Patients underwent contrast-enhanced studies and routine T1-weighted sagittal imaging in the prone position before and after NAC. Fast spin echo images with fat suppression $(\mathrm{TR} / \mathrm{TE}=467 /$ $10.0 \mathrm{~ms}$, slice thickness $5.0 \mathrm{~mm}$, interslice gap $2.5 \mathrm{~mm}$, FOV $24 \mathrm{~cm}$, matrix 256x192) were obtained both prior to intravenous administration of gadopentetate dimeglumine and then 10 times over the following $5 \mathrm{~min}$. We measured the minor axis diameter of the enhancing region for each area adjacent to the breast tumor during the early phase of the contrast-enhanced study (Fig. 1c). We employed the greatest minor axis diameter value in the analysis. Two radiologists (Y.M. and N.H.) with 11-16 years experience in breast MRI analyzed the MRI findings without information from any other imaging modality.

Statistical analysis. We tested differences in the frequency of IDS using the $\chi^{2}$ test. A variable with a P-value of $<0.05$ was considered a potential predictor for the presence of IDS. Multiple logistic regression analyses (forward stepwise) were performed to control the influence of potential confounding factors; a variable with a P-value $<0.05$ was regarded as an independent predictor for IDS using this model.

\section{Results}

Correlation between the findings of the three modalities and the histopathologic results are presented in Tables I and II, 
Table II. Correlation between findings of US and MRI and pathologic analysis of IDS.

\begin{tabular}{lcccccc}
\hline & & & & \multicolumn{2}{c}{ P-value } \\
\cline { 5 - 6 } $\begin{array}{l}\text { Max diameter } \\
(\mathrm{mm})^{\mathrm{a}}\end{array}$ & $0-0.9$ & $1.0-1.9$ & $2.0-2.9$ & $\leq 3.0$ & Univariate & Multivariate \\
\hline Pre-NAC-US & 9 & 11 & 7 & 30 & $<0.01$ & $<0.01$ \\
After-NAC-US & 8 & 16 & 17 & 16 & $<0.01$ & 0.15 (NS) \\
Pre-NAC-MRI & 4 & 16 & 4 & 33 & $<0.01$ & 0.83 (NS) \\
After-NAC-MRI & 12 & 10 & 17 & 18 & $<0.01$ & 0.73 (NS) \\
\hline
\end{tabular}

${ }^{a}$ Maximal diameter of hypoechoic structure on US and enhanced area on MRI. Numbers represent the number of breast tissue areas with IDS (57 areas in total).

Table III. Accuracy of MMG and US.

\begin{tabular}{lcc}
\hline & MMG & US \\
\hline Sensitivity & 57.9 & 52.6 \\
Specificity & 95.3 & 96.3 \\
PPV & 86.8 & 88.2 \\
NPV & 81.0 & 79.2 \\
OA & 82.3 & 81.1 \\
\hline
\end{tabular}

PPV, positive predictive value; NPV, negative predictive value; OA, overall accuracy. Cut-off value for diameter of hypoechoic area is $3.0 \mathrm{~mm}$ for US.

respectively. The results of MMG after NAC have been omitted because the results of MMG before and after NAC were identical. Although univariate analysis revealed statistical significance for all modalities both before and after NAC, multivariate analysis indicated that MMG and US before NAC are predictors of IDS. The predictive abilities of MMG and US prior to NAC are presented in Table III.

\section{Discussion}

Several investigators report the use of imaging techniques that aim to detect IDS and avoid residual cancer cells in conservative breast surgery (13-17). The sensitivity of MMG, US and MRI in these studies ranged from 21.1-55\%, 20.6$89 \%$ and $66.7-93 \%$, respectively, while specificity ranged from $85.7-100 \%, 76-100 \%$ and $60-90 \%$, and accuracy ranged from $42.3-72 \%, 50-85 \%$ and $65.6-92 \%$. The breast cancer patients in these previous reports had not received NAC. In the present study, we found the predictive ability of MMG and US to be equal to the results of previous studies, with or without NAC. Imaging modalities were of use in detecting IDS; however, previous reports found the predictive ability of these modalities to vary widely. Previous investigators defined the criteria for IDS to be pleomorphic or heterogeneous calcification on MMG $(14,15,17)$, small hypoechoic area on US $(15,17)$, and enhanced area on MRI (14-17).
Enhancement around the main tumor is considered to be IDS on MRI; however, according to previous reports, MRI possesses low specificity despite its high sensitivity $(13,14)$. We considered that the criteria employed in the previous studies contain inconsistencies that require resolution. Multivariate analysis excluded the MRI findings as a predictive variable of IDS in the present study. We consider that MRI is useful in the diagnosis of daughter lesions, as mentioned in previous reports, but is not useful in the detection of IDS (27-30): the diameters of the daughter lesions are detectable but the IDS is too small to be distinguished from normallyenhancing breast tissue. The hypoechoic area around the main tumor on US represents not only IDS but also normal structure (31). Therefore, hypoechoic findings on US require an objective index for the prediction of IDS. We demonstrated that $3.0 \mathrm{~mm}$ is an adequate cut-off value to identify the existence of IDS on the basis of size of the hypoechoic structure. Therefore, US was reliable in the preoperative assessment of breast cancer in the current study.

Findings of a large hypoechoic area $(>3 \mathrm{~mm})$ on US and microcalcification on MMG both suggest the existence of IDS with an equally high positive predictive value. For this reason, breast tissue that contains these findings is contraindicative for preservation. In contrast, the sensitivity of these findings is too low to use as a basis for tissue conservation. As an alternative method, breast cancer that is not accompanied by a large hypoechoic area or microcalcification should be resected with a $1-$ to $2-\mathrm{cm}$ safety margin in conservative surgery, followed by simultaneous rapid pathological analysis during surgery. Additional resection of breast tissue is required when pathological analysis reveals cancer cells near the surgical margin.

In the current study, we demonstrated that MMG and US enable the reliable prediction of IDS following NAC, thereby removing any uncertainty regarding the value of these modalities. MMG and US therefore provide a reliable basis for BCT, with or without NAC.

\section{References}

1. Ogawa $\mathrm{Y}$, Nishioka A, Inomata $\mathrm{T}$, Tsuboi N, Hayase $\mathrm{N}$, Fukumoto M, Yoshida S, Tohchika N, Tanaka Y and Kumon M: Conservation treatment intensified with tamoxifen and CAF chemotherapy for subareolar breast cancers. Oncol Rep 5: 1337-1341, 1998. 
2. Nishioka A, Ogawa Y, Hamada N, Terashima M, Inomata T and Yoshida S: Analysis of radiation pneumonitis and radiationinduced lung fibrosis in breast cancer patients after breast conservation treatment. Oncol Rep 6: 513-517, 1999.

3. Ogawa Y, Nishioka A, Inomata T, Yokota N, Sasaki T, Terashima M, Yoshida S, Tanaka Y and Tohchika N: Conservation treatment intensified with tamoxifen and CAF chemotherapy without axillary dissection for early breast cancer patients with clinically-negative axillary nodes. Oncol Rep 6: 801-805, 1999.

4. Ogawa Y, Nishioka A, Inomata T, Ohnishi T, Kariya S, Terashima M, Yoshida S, Tohchika N, Tanaka Y and Kumon M: Conservation treatment intensified with an anti-estrogen agent and CAF chemotherapy for stage I and II breast cancer. Oncol Rep 7: 479-484, 2000.

5. Terashima M, Ogawa $Y$, Kariya S, Inomata T, Nishioka A, Shimizu K, Yamanishi T, Tanaka Y, Tochika N and Yoshida S: Breast-conservation treatment for patients with ductal carcinoma in situ. Oncol Rep 7: 1247-1252, 2000.

6. Hamada N, Ogawa Y, Nishioka A, Kariya S, Terashima M, Yoshida S, Tochika N, Tanaka Y, Kumon M and Inomata T: Breast-conservation treatment for bilateral breast cancer in five Japanese women. Oncol Rep 9: 469-474, 2002.

7. Veronesi U, Cascinelli N, Mariani L, Greco M, Saccozzi R, Luini A, Aguilar M and Marubini E: Twenty-year follow-up of a randomized study comparing breast-conserving surgery with radical mastectomy for early breast cancer. N Engl J Med 347: $1227-1232,2002$

8. Hamada N, Ogawa Y, Nishioka A, Kariya S and Yoshida S: Primary toremifene treatment for elderly post-menopausal women with breast cancer. Oncol Rep 11: 365-370, 2004.

9. Fisher B, Anderson S, Bryant J, Margolese RG, Deutsch M, Fisher ER, Jeong JH and Wolmark N: Twenty-year follow-up of a randomized trial comparing total mastectomy, lumpectomy and lumpectomy plus irradiation for the treatment of invasive breast cancer. N Engl J Med 347: 1233-1241, 2002.

10. Murata Y, Ogawa Y, Yoshida S, Kubota K, Itoh S, Fukumoto M, Nishioka A, Moriki T, Maeda H and Tanaka Y: Utility of initial MRI for predicting extent of residual disease after neoadjuvant chemotherapy: analysis of 70 breast cancer patients. Oncol Rep 12: $1257-1262,2004$

11. Lindley R, Bulman A, Parsons P, Phillips R, Henry K and Ellis H: Histologic features predictive of an increased risk of early local recurrence after treatment of breast cancer by local tumor excision and radical radiotherapy. Surgery 105: 13-20, 1989.

12. Holland R, Connolly JL, Gelman R, Mravunac M, Hendriks JH, Verbeek AL, Schnitt SJ, Silver B, Boyages J and Harris JR: The presence of an extensive intraductal component following a limited excision correlates with prominent residual disease in the remainder of the breast. J Clin Oncol 8: 113-118, 1990.

13. Hiramatsu H, Enomoto K, Ikeda T, Mukai M, Furukawa J, Kikuchi K, Oshio K, Hiraoka N, Kitajima M and Hiramatsu K: The role of contrast enhanced high resolution MRI in the surgical planning of breast cancer. Breast Cancer 25: 285-290, 1997.

14. Satake H, Shimamoto K, Sawaki A, Niimi R, Ando Y, Ishiguchi T, Ishigaki T, Yamakawa K, Nagasaka T and Funahashi H: Role of ultrasonography in the detection of intraductal spread of breast cancer: correlation with pathologic findings, mammography and MR imaging. Eur Radiol 10: 1726-1732, 2000.

15. Tamaki $Y$, Akashi-Tanaka S, Ishida T, Uematsu T, Sawai $Y$, Kusama M, Nakamura S, Hisamatsu K, Tanji Y, Sato Y and Matsuura N: 3D imaging of intraductal spread of breast cancer and its clinical application for navigation surgery. Breast Cancer 9: 289-295, 2002 .

16. Hata T, Takahashi H, Watanabe K, Takahashi M, Taguchi K, Itoh T and Todo S: Magnetic resonance imaging for preoperative evaluation of breast cancer: a comparative study with mammography and ultrasonography. J Am Coll Surg 198: 190-197, 2004.
17. Komatsu S, Lee CJ, Hosokawa Y, Ichikawa D, Hamashima T, Shirono K, Okabe H, Kurioka H and Oka T: Comparison of intraductal spread on dynamic contrast-enhanced MRI with clinicopathologic features in breast cancer. Jpn J Clin Oncol 34: 515-518, 2004.

18. Wolmark N, Wang J, Mamounas E, Bryant J and Fisher B: Preoperative chemotherapy in patients with operable breast cancer: nine-year results from National Surgical Adjuvant Breast and Bowel Project B-18. J Natl Cancer Inst Monogr 30: 96-102, 2001.

19. Van der Hage JA, van de Velde CJ, Julien JP, Tubiana-Hulin M, Vandervelden C and Duchateau L: Preoperative chemotherapy in primary operable breast cancer: results from the European Organization for Research and Treatment of Cancer trial 10902. J Clin Oncol 19: 4224-4237, 2001.

20. Kariya S, Ogawa Y, Nishioka A and Yoshida S: Docetaxelcisplatin combined chemotherapy in Japanese patients with anthracycline-pretreated advanced breast cancer. Oncol Rep 9: 1345-1349, 2002.

21. Ogawa Y, Nishioka A, Nishigawa T, Kubota K, Kariya S, Yoshida S, Tanaka Y, Moriki T and Tochika N: Thin-section CT evaluation and pathologic correlation of therapeutic effect of neoadjuvant chemotherapy for axillary lymph nodes of clinically node-positive breast cancer patients. Oncol Rep 10: 985-989, 2003.

22. Ogawa Y, Nishioka A, Kubota K, Kariya S, Yoshida S, Maeda H, Tanaka Y, Moriki T and Tochika N: CT findings of breast cancer with clinically complete response following neoadjuvant chemotherapy-histological correlation. Oncol Rep 10: 1411-1415, 2003.

23. Kariya S, Ogawa Y, Nishioka A, Moriki T, Ohnishi T, Ito S, Murata Y and Yoshida S: Relationship between hormonal receptors, HER-2, p53 protein, Bcl-2 and MIB-1 status and the antitumor effects of neoadjuvant anthracycline-based chemotherapy in invasive breast cancer patients. Radiat Med 23: 189-194, 2005.

24. Tanaka Y, Maeda H, Ogawa Y, Nishioka A, Itoh S, Kubota K, Ue H, Nakatani K and Sasaguri S: Sentinel node biopsy in breast cancer patients treated with neoadjuvant chemotherapy. Oncol Rep 15: 927-931, 2006.

25. Okawa T, Kodama H, Akiyama F, et al: Guideline for the breast conservative therapy. Japanese Society of Breast Cancer, Kyoiku-koho-sya, 1999.

26. American College of Radiology: Breast imaging and reporting system. In: Mammography. 4th edition. American College of Radiology, Reston, VA, pp61-128, 2003.

27. Orel SG, Schnall MD, Powell CM, Hochman MG, Solin LJ, Fowble BL, Torosian MH and Rosato EF: Staging of suspected breast cancer: effect of MR imaging and MR-guided biopsy. Radiology 196: 115-122, 1995

28. Esserman L, Hylton N, Yassa L, Barclay J, Frankel S and Sickles E: Utility of magnetic resonance imaging in the management of breast cancer: evidence for improved preoperative staging. J Clin Oncol 17: 110-119, 1999.

29. Liberman L, Morris EA, Dershaw DD, Abramson AF and Tan LK: MR imaging of the ipsilateral breast in women with percutaneously proven breast cancer. AJR 180: 901-910, 2003.

30. Berg WA, Gutierrez L, NessAiver MS, Carter WB, Bhargavan M, Lewis RS and Ioffe OB: Diagnostic accuracy of mammography, clinical examination, US and MR imaging in preoperative assessment of breast cancer. Radiology 233: 830-849, 2004.

31. American College of Radiology: Breast imaging and reporting system. In: Mammography. 4th edition. American College of Radiology, Reston, VA, pp5-8, 2003. 